\title{
Buenas prácticas de manufactura para procesamiento y conservación de vegetales
}

\section{Good manufacturing practices for vegetable processing and preservation}

\section{Boas práticas de fabricação para processamento e conservação de vegetais}

\author{
Guzmán Cupaja Diego Fernando ${ }^{1}$ y Ayza Yamir Urbina Angarita ${ }^{2}$ \\ ${ }^{1}$ Ingeniero Agroindustrial, Universidad de los Llanos y \\ ${ }^{2}$ Ingeniera Industrial, MSc, Docente Universidad de los Llanos \\ aurbina@unillanos.edu.co
}

Recibido 20 de enero 2021, Aceptado 23 de abril 2021

\section{RESUMEN}

El presente trabajo se llevó a cabo en una empresa que tiene como actividad el procesamiento y conservación de frutas, legumbres, hortalizas y tubérculos para consumo humano, mediante el proceso de deshidratación; esta empresa se encuentra ubicada en la ciudad de Villavicencio (Colombia). El objetivo fue diseñar el Manual de Buenas Prácticas de Manufactura (BPM), el cual comprende un conjunto de lineamientos que permite tener un sistema actual documental basado en los requisitos de la normatividad vigente, para lograr este propósito se estructuró de la siguiente manera: Fase de diagnóstico mediante la inspección visual a las instalaciones de la planta y entrevistas al personal de que trabaja en la empresa, para ello se creó una lista de chequeo basada en la reglamentación vigente, obteniéndose un porcentaje de cumplimiento de $74 \%$. A partir de este resultado, se elaboró un plan de acción identificando las no conformidades, evidenciándose la necesidad de la implementación de un manual de BPM. La segunda fase fue la documental: A partir de los resultados de inspección se diseñó el Manual de Buenas Prácticas de Manufactura, el cual incluye: el plan de saneamiento, capacitación de personal de manipulador de alimentos y sistema de control. La tercera fase fue la de implementación: se construyó un documento de plan de implementación que le permite a la empresa realizar una mejora continua de las actividades faltantes y se estructuró de la siguiente manera: objetivo general, tareas propuestas, cronograma 
de actividades y asignación de responsables. Finalmente, el Manual de Buenas Prácticas de Manufactura, la documentación y el plan de implementación que se diseñaron, permitieron a la empresa garantizar el cumplimiento de los requerimientos establecidos por la normatividad nacional e internacional, lo que conlleva, a la elaboración de productos en condiciones sanitarias y de calidad adecuadas que cumplan con las expectativas de los clientes.

Palabras clave: Inocuidad, alimentos secos, plan de saneamiento básico, manipulación.

\section{ABSTRACT}

The present work was carried out in a company whose activity is the processing and preservation of fruits, legumes, vegetables and tubers for human consumption, through the dehydration process; This company is located in the city of Villavicencio (Colombia). The objective was to design the Manual of Good Manufacturing Practices (GMP), which comprises a set of guidelines that allows to have a current documentary system based on the requirements of current regulations, to achieve this purpose it was structured as follows : Diagnostic phase by means of a visual inspection of the plant facilities and interviews with the personnel who work in the company, for which a checklist was created based on current regulations, obtaining a compliance percentage of $74 \%$. Based on this result, an action plan was drawn up identifying the non-conformities, evidencing the need for the implementation of a BPM manual. The second phase was the documentary one: Based on the inspection results, the Good Manufacturing Practices Manual was designed, which includes: the sanitation plan, training of food handler personnel and the control system. The third phase was the implementation phase: an implementation plan document was built that allows the company to continuously improve the missing activities and was structured as follows: general objective, proposed tasks, activity schedule and assignment of responsible. Finally, the Manual of Good Manufacturing Practices, the documentation and the implementation plan that were designed, allowed the company to guarantee compliance with the requirements established by national 
and international regulations, which leads to the elaboration of products in conditions quality and sanitary facilities that meet customer expectations.

Keywords: Safety, dry food, basic sanitation plan, handling.

\section{RESUMO}

O presente trabalho foi realizado em uma empresa cuja atividade é o beneficiamento e conservação de frutas, legumes, verduras e tubérculos para consumo humano, através do processo de desidratação; Esta empresa está localizada na cidade de Villavicencio (Colômbia). O objetivo foi elaborar o Manual de Boas Práticas de Fabricação (BPF), que compreende um conjunto de diretrizes que permite ter um sistema documental atualizado com base nos requisitos da, para regulamentos atuais atingir esse objetivo foi estruturado da seguinte forma: Diagnóstico fase por meio de inspeção visual das instalações da fábrica e entrevistas com o pessoal que trabalha na empresa, para a qual foi elaborado um checklist com base na regulamentação em vigor, obtendo-se um percentual de conformidade de $74 \%$. A partir desse resultado, foi elaborado um plano de ação identificando as não conformidades, evidenciando a necessidade de implantação de um manual de BPM. A segunda fase foi documental: Com base nos resultados da fiscalização, foi elaborado o Manual de Boas Práticas de Fabricação, que contempla: o plano de saneamento, o treinamento dos manipuladores de alimentos e o sistema de controle. A terceira fase foi a fase de implementação: foi construído um documento de plano de implementação que permite à empresa melhorar continuamente as atividades em falta e foi estruturado da seguinte forma: objetivo geral, tarefas propostas, cronograma de atividades e atribuição de responsáveis. Por fim, o Manual de Boas Práticas de Fabricação, a documentação e o plano de implantação que foram traçados, permitiram à empresa garantir o cumprimento dos requisitos estabelecidos pela regulamentação nacional e internacional, o que leva à elaboração de produtos em condições de qualidade e instalações sanitárias que atendam expectativas do cliente.

Palavras-chave: Segurança, alimento seco, plano de saneamento básico, manejo. 


\section{INTRODUCCIÓN}

Actualmente, las enfermedades transmitidas por alimentos (ETA) se caracterizan por ser un problema de salud pública a nivel mundial ya que generan un riesgo muy alto sobre la salud de las personas, este factor afecta negativamente la economía de países y empresas en su productividad, además, de alterar el comercio y la confianza de los consumidores (Rodríguez et al., 2015).

Por otra parte, el proceso de globalización ha provocado el aumento de la comercialización de alimentos a nivel nacional e internacional, consigo a traído beneficios sociales y económicos, pero también ha facilitado la propagación de enfermedades en el mundo, debido a la deficiencia en el proceso de elaboración, manipulación, conservación, transporte, distribución o comercialización Es por esto que todos los fabricantes de alimentos se han dado cuenta de la importancia de asegurar la calidad de sus productos, desde la producción primaria hasta el consumo final, además de brindar un alto nivel de satisfacción a los clientes (Ayuso y Castillo, 2017).

Por lo anterior, se ve la necesidad de la implementación de Buenas Prácticas de Manufactura y el uso de las normas y decretos que le competen y que permiten que los productos cumplan con los requerimientos a nivel de empresa y de cliente. Para lograr este propósito las empresas deben contar con este sistema de aseguramiento de calidad que se basa en principios básicos y prácticas de higiene en la manipulación, preparación, elaboración, envasado, almacenamiento, transporte y distribución de alimentos para el consumo humano, y garantizar que los productos en cada una de las etapas de la cadena productiva cumplan con las condiciones sanitarias adecuadas, de manera que, se disminuyan los riesgos inherentes a la producción y al producto. Las ventajas del uso de BPM, generan no solo ventajas en la salud, sino que también, los empresarios se ven beneficiados en términos de reducción de pérdidas de producto, costos, entre otros, así mismo, esto mejora el posicionamiento de sus productos, debido al reconocimiento de sus atributos (INPEC, 2017). 


\section{BUENAS PRÁCTICAS DE MANUFACTURA}

Las Buenas Prácticas de Manufactura (BPM), se han definido como "un conjunto de principios básicos y prácticos generales de higiene en la manipulación, preparación, elaboración, envasado, almacenado, transporte y distribución de alimentos para consumo humano; con el objeto de garantizar que los productos en cada una de las operaciones mencionadas cumplan con las condiciones sanitarias adecuadas, de modo que se disminuyan los riesgos inherentes a la producción" (Albarracín y Carrascal, 2005).

La FAO, (2011) considera las BPM como un conjunto de directrices creado y establecido con el único fin de garantizar un entorno laboral inocuo y seguro para evitar la contaminación de los alimentos a lo largo de las diferentes etapas del proceso productivo, al igual que durante su producción y comercialización. La inocuidad es entendida como la garantía de que un alimento para consumo humano no cause daño al consumidor al momento de ser ingerido o de acuerdo con el uso al que sea destinado.

Dentro de estas directrices, se incluyen normas de comportamiento del personal manipulador de alimentos en el área de trabajo, la adecuada distribución de las zonas de trabajo y el uso de agua y desinfectantes, entre otros. En otras palabras, las BPM son una herramienta básica para obtener productos seguros para el consumo humano, gracias a la inclusión de protocolos basados en las prácticas higiénicas y la manipulación de los alimentos. Por lo tanto, son útiles para el diseño y el funcionamiento de los establecimientos encargados de transformar, manipular y comercializar alimentos, así como para el desarrollo adecuado de los procesos de elaboración de alimentos.

Las Buenas Prácticas de Manufactura (BPM) están determinadas en Colombia por la Resolución 2674 de 2013, en el artículo 1 establece: " los requisitos sanitarios que deben cumplir las personas naturales y/o jurídicas que ejercen actividades de fabricación, procesamiento, preparación, envase, almacenamiento, transporte, distribución y comercialización de alimentos y materias primas de alimentos y los 
requisitos para la notificación, permiso o registro sanitario de los alimentos, según el riesgo en salud pública, con el fin de proteger la vida y la salud de las personas" y el ente regulador de vigilancia y control es el Instituto Nacional de Vigilancia de Medicamentos y Alimentos (INVIMA) (MINSALUD, 2013). El procesamiento y conservación de frutas, legumbres, hortalizas y tubérculos para consumo humano mediante a se clasifica como un producto de bajo riesgo según la Resolución 719 de 2015, por lo que, requieren de permisos sanitarios para su comercialización y consumo (MINSALUD, 2015). Las empresas que se dedican a esta actividad necesitan implementar las Buenas Prácticas de Manufactura (BPM), teniendo en cuenta los requisitos de Resolución No. 2674 de 2013, con el fin de mejorar los procesos productivos y de competitividad, obteniendo productos inocuos y de calidad, que le permitan un mejor posicionamiento en el mercado.

Actualmente, las BPM se establecen como un requisito básico para poder aplicar un sistema de Análisis de Peligros y Puntos Críticos de Control, (APPCC) ○ Hazard Analysis and Critical Control Points, (HACCP) que son las siglas en inglés dentro de un programa de Gestión de la Calidad o en un Sistema de Calidad ISO ${ }^{10}$. El sistema surge como una política de globalización y estandarización que permite establecer cada vez más requisitos para el consumo de alimentos. Su origen se establece en el año 1959 en la Agencia Aeroespacial de Estados Unidos de América, NASA, como un programa creado para garantizar la inocuidad de los alimentos consumidos por los astronautas en el espacio; siendo la empresa Pillsbury Co. quien introdujo tal sistema de control para favorecer dichas garantías de calidad e inocuidad. Luego, en 1993 la Comisión Codex Alimentarius aprueba unas directrices para la aplicación del sistema HACCP y es a partir del año 2005 que se publica la norma ISO 22000, en la cual se establece el Sistema de Gestión de Inocuidad Alimentaria basado en ISO 9001 y el sistema HACCP (Mena, 2014).

\section{PLAN DE SANEAMIENTO}

Según lo establecido en la Resolución 2074 de 2013 en el artículo 26, todos los establecimientos destinados a la fabricación, procesamiento, envase y almacenamiento de alimentos debe implantar y desarrollar un plan de saneamiento, 
en donde se establecen de manera clara sus objetivos y los procedimientos requeridos para disminuir los riesgos de contaminación de los alimentos. Este plan debe presentarse por escrito y estar a disposición de la autoridad sanitaria competente; incluyendo como mínimo los procedimientos, cronogramas, registros, listas de chequeo y responsables, como mínimo, de los siguientes programas: 1. Limpieza y desinfección, 2. Residuos sólidos, 3. Control de plagas, 4. Abastecimiento o suministro de agua potable y 5 . Programas complementarios.

\section{Programa de limpieza y desinfección}

Se especifican las condiciones necesarias de limpieza y desinfección particulares del proceso y del producto de la empresa. Se deben registrar por escrito, todos y cada uno de los procedimientos de la planta, al igual que las fichas técnicas de los productos o substancias utilizadas, marca, concentración, preparación, forma de uso, periodicidad (cronograma) y demás elementos o equipos necesarios para desarrollar las tareas de limpieza y desinfección.

\section{Programa de manejo de residuos}

La planta debe contar con las instalaciones, elementos, áreas, recursos y procedimientos necesarios que permitan garantizar un sistema eficiente y adecuado de recolección, conducción, manejo, almacenamiento interno, clasificación,

transporte y disposición de los residuos sólidos generados a lo largo del proceso. Las actividades mencionadas deben realizarse teniendo en cuenta las normas vigentes de higiene, salud y seguridad en el trabajo con el único objetivo de evitar la contaminación de los alimentos, maquinaria y equipos, áreas de proceso o circundantes, dependencias y el impacto negativo sobre el medio ambiente.

\section{Programa de manejo o control de plagas}

Las plagas deben considerarse dentro de un programa específico el cual involucra el concepto de control integral (Manejo Integrado de Plagas, MIP); es decir, que se deben establecer medidas de aplicación sistemática y armónica de las diversas 
medidas de control, con énfasis en la erradicación y otras adicionales de orden preventivo.

\section{Programa de abastecimiento de agua potable}

Según lo establecido en la Resolución 2674 de 2013, todos los tipos de establecimientos mencionados están obligados a documentar un este programa, incluyendo: fuente de captación, tratamientos, manejo, diseño y capacidad de almacenamiento, distribución, mantenimiento, limpieza y desinfección del sistema hidráulico y tanques de almacenamiento. La implementación de este programa debe garantizar el cumplimiento de las condiciones fisicoquímicas y microbiológicas vigentes para el agua de consumo humano y procesos en la industria de alimentos; así como los registros físicos que brinden soporte a su estricto cumplimiento.

\section{Programas complementarios}

Los considerados de la Resolución 2674 de 2013 son: capacitación, mantenimiento de instalaciones y equipos, plan de trazabilidad, análisis de peligros y puntos críticos de control (APPCC) y plan de muestreo, además es necesario tener en cuenta:

1) Ubicación de las instalaciones, su estructura física, la distribución de planta y de equipos. 2) Abastecimiento de agua, desagües y eliminación de desechos, la higiene del personal, limpieza y desinfección de las instalaciones. 3) Aspectos operativos, verificación y manejo y almacenamiento de materias primas, aditivos alimentarios y envases, rechazo o descarte de producto no- conforme y transporte y 4) Requisitos sanitarios y ambientales

\section{ANÁLISIS DE LAS VENTAJAS DE IMPLEMENTAR LAS BPM}

Implementar BPM en una empresa ofrece múltiples ventajas, tanto para la organización como para los clientes. por ejemplo, contribuye a la mejora de los procesos productivos y de calidad, reduce los tiempos de ejecución, ayuda a establecer los puntos críticos y los "cuellos de botella" del proceso, mejora las comunicaciones, permite realizar una trazabilidad efectiva, mejora la higiene de los productos y procesos, aumenta la vida útil del producto, mejora la imagen 
empresarial, estandariza el sistema de inocuidad en la producción, garantiza una planta que cumple con la normatividad vigente, crea nuevas posibilidades de productos y amplios mercados.

La microempresa agroindustrial llanera en la cual se analizaron la aplicación de las BPM se denomina Agroacecar SAS, siendo su actividad económica principal el procesamiento y conservación de frutas, legumbres, hortalizas y tubérculos para consumo humano mediante la técnica de deshidratación en horno eléctrico mediante convección forzada de aire caliente. Su misión es transforma materias primas del agro colombiano en productos alimenticios de alta calidad, 100\% saludables para ser incorporados en cualquier tipo de dieta alimenticia. Presta los servicios de producción de fruta deshidratada, maquila de productos mercado nacional e internacional, pruebas de deshidratación (frutas) y visitas académicas.

La planta tiene un distribuida en un primer y único nivel de alrededor de $7 \times 9 \mathrm{~m}$. La producción se desarrolla en $U$, teniendo 3 tiempos diferentes para entrada de materia prima, salida de producto terminado y salida de residuos sólidos (incluye producto no-conforme) (Figura 1). La producción de frutas deshidratadas en este caso sigue su línea de producción de forma en u, con una misma entrada de materia prima en diferentes tiempos, para evitar la contaminación cruzada, seguidamente se evidencia en su distribución de planta las diferentes áreas establecidas, como se puede evidenciar en la Figura 1. La normatividad legal vigente para llevar a cabo el análisis de Buenas Prácticas de Manufactura para la empresa Agroacecar SAS, se detalla en la Tabla 1.

\section{RESULTADOS Y PROCEDIMIENTO EN EL ANÁLISIS DE LA BMP EN EL EMPRESA AGROACECAR SAS}

\section{Diagnóstico}

Se realizó un diagnóstico inicial sobre las condiciones higiénico-sanitarias según la normativa colombiana vigente, se diseñó una lista de chequeo basada en la Resolución 2674 de 2013, realizando entrevistas al personal y entrevistas directas con todos los responsables del proceso, posteriormente, se llevó a cabo la 
cuantificación del porcentaje de cumplimiento. Para el diagnóstico higiénicosanitario, se elaboró una escala de valoración como se especifica en la Tabla 2.

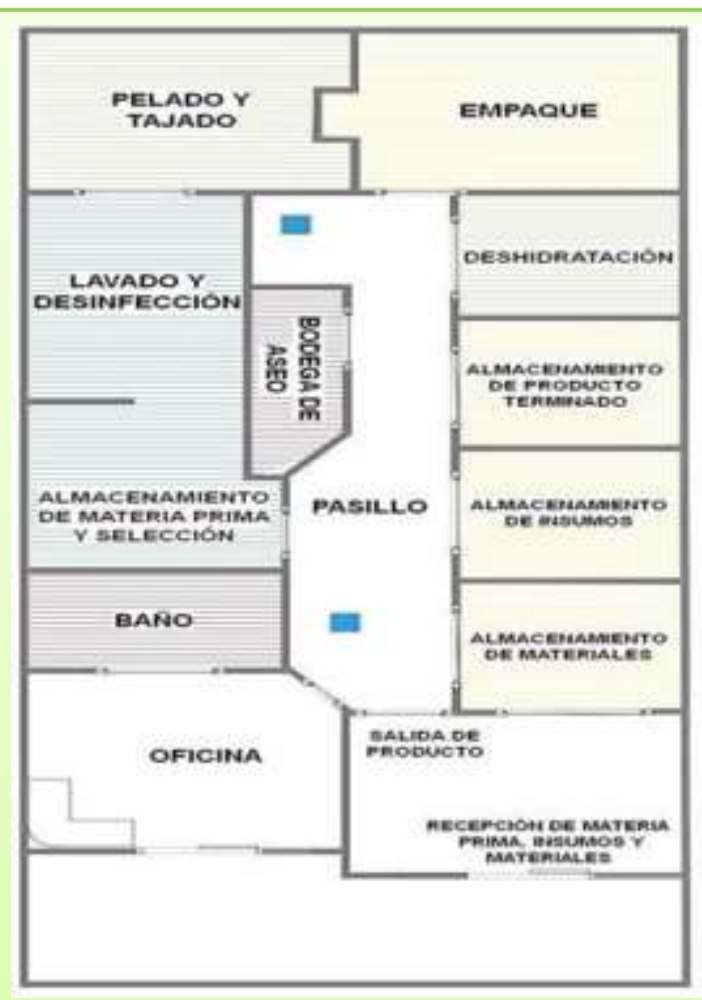

Figura 1. Plano de la planta de producción de Agroacecar SAS., especificando cada una de las áreas del proceso. Fuente: Agroacecar SAS.

Se verificaron los siguientes aspectos de acuerdo con la resolución 2674 de 2013 de la siguiente manera:

1. Aseguramiento y control de calidad: Hace referencia a todo lo relacionado con políticas de calidad, procedimientos, fichas técnicas de materiales, manuales, guías e instructivos, sobre los equipos, procesos, condiciones de almacenamiento y distribución, procesos de control de calidad, y otros aspectos.

2. Instalaciones físicas y sanitarias: se basa en la estructura de la planta en cuanto a construcción y diseño, los accesos y sus alrededores; servicios de lavamanos, inodoros, dotación de elementos (jabón, toallas, papel, caneca), vestidores, área social. 
Tabla 1. Normatividad legal para el análisis de Buenas Prácticas de Manufactura (BPM)

\begin{tabular}{|c|c|c|}
\hline Norma & Ente que reglamanta & Objeto \\
\hline $\begin{array}{l}\text { Ley } 9 \text { de } \\
1979\end{array}$ & $\begin{array}{l}\text { Congreso de } \\
\text { Colombia }\end{array}$ & Por la cual se dictan medidas sanitarias \\
\hline $\begin{array}{l}\text { Decreto } \\
1575 \text { de } \\
2007\end{array}$ & $\begin{array}{l}\text { Ministerio de Salud y } \\
\text { Protección Social }\end{array}$ & $\begin{array}{l}\text { Por el cual se establece el sistema para la } \\
\text { protección y control de la calidad del agua para } \\
\text { consumo humano }\end{array}$ \\
\hline $\begin{array}{l}\text { Resolución } \\
5109 \text { de } \\
2005\end{array}$ & $\begin{array}{l}\text { Ministerio de Salud y } \\
\text { Protección Social }\end{array}$ & $\begin{array}{l}\text { Por el cual se establece el } \\
\text { reglamento técnico sobre los requisitos de } \\
\text { rotulado o etiquetado que deben cumplir los } \\
\text { alimentos envasados y materias primas de } \\
\text { alimentos para consumo humano }\end{array}$ \\
\hline $\begin{array}{l}\text { Resolución } \\
2115 \text { de } \\
2007\end{array}$ & $\begin{array}{l}\text { Ministerio de Salud y } \\
\text { Protección Social y } \\
\text { Ministerio de } \\
\text { Ambiente, } \\
\text { Vivienda y } \\
\text { Desarrollo Territorial }\end{array}$ & $\begin{array}{l}\text { Por medio de la cual se señalan características, } \\
\text { instrumentos básicos y frecuencias del sistema } \\
\text { de control y vigilancia para la calidad del agua } \\
\text { para consumo humano. }\end{array}$ \\
\hline $\begin{array}{l}\text { Resolución } \\
333 \text { de } \\
2011\end{array}$ & $\begin{array}{l}\text { Ministerio de Salud y } \\
\text { Protección Social }\end{array}$ & $\begin{array}{l}\text { Por la cual se establece el reglamento técnico } \\
\text { sobre los requisitos de rotulado o etiquetado } \\
\text { nutricional que deben cumplir los alimentos } \\
\text { envasados para consumo humano. }\end{array}$ \\
\hline Resolución & Ministerio de Salud y & $\begin{array}{l}\text { Por medio de la cual se expide el reglamento } \\
\text { técnico sobre los requisitos sanitarios que } \\
\text { deben cumplir los materiales, objetos, }\end{array}$ \\
\hline 2012 & Protección Social & $\begin{array}{l}\text { envases y equipamientos destinados a entrar en } \\
\text { contacto con alimentos y bebidas para consumo } \\
\text { humano. }\end{array}$ \\
\hline $\begin{array}{l}\text { Resolución } \\
4142 \text { de } \\
2012\end{array}$ & $\begin{array}{l}\text { Ministerio de Salud y } \\
\text { Protección Social }\end{array}$ & $\begin{array}{l}\text { Por la cual se establece el reglamento técnico } \\
\text { sobre los requisitos sanitarios que deben } \\
\text { cumplir los materiales, objetos, envases y } \\
\text { equipamientos metálicos destinados a entrar en } \\
\text { contacto con alimentos y bebidas para consumo } \\
\text { humano en el territorio nacional. }\end{array}$ \\
\hline $\begin{array}{l}\text { Resolución } \\
835 \text { de } 2013\end{array}$ & $\begin{array}{l}\text { Ministerio de Salud y } \\
\text { Protección Social }\end{array}$ & $\begin{array}{l}\text { Por la cual se establece el reglamento técnico } \\
\text { sobre los requisitos sanitarios que deben } \\
\text { cumplir los materiales, objetos, envases y } \\
\text { equipamientos de vidrios y cerámicas } \\
\text { destinados a estar en contacto con alimentos y } \\
\text { bebidas para el consumo humano. }\end{array}$ \\
\hline $\begin{array}{l}\text { Resolución } \\
2674 \text { de } \\
2013\end{array}$ & $\begin{array}{l}\text { Ministerio de Salud y } \\
\text { Protección Social }\end{array}$ & $\begin{array}{l}\text { Por la cual se reglamenta el artículo } 126 \text { del } \\
\text { Decreto-ley } 019 \text { de } 2012 \text { y se dictan otras } \\
\text { disposiciones. }\end{array}$ \\
\hline
\end{tabular}


Tabla 2. Escala de valoración de la lista de chequeo.

\begin{tabular}{lcc}
\hline \multicolumn{1}{c}{ Parámetro } & Calificación & Valor de calificación \\
\hline Cumple completamente & 2 & 5 \\
Cumple parcialmente & 1 & 4 \\
No cumple & 0 & 3 \\
No aplica & NA & 2 \\
No observado & NO & 1 \\
\hline
\end{tabular}

Nota: Se asignaron valores a la calificación para realizar el análisis de los resultados.

Fuente: Los autores

3. Personal manipulador de alimentos: Se fundamenta en las prácticas higiénicas y medidas de protección, educación y capacitación del personal.

4. Condiciones de saneamiento: Se basa en el plan de saneamiento básico que comprende: abastecimiento de agua potable, manejo de residuos líquidos y disposición de residuos sólidos, limpieza y desinfección, control de plagas (artrópodos, roedores, aves).

5. Condiciones de proceso y fabricación: tiene en cuenta los siguientes aspectos: equipos y utensilios, higiene locativa de la sala de proceso, materias primas e insumos, envases, operaciones de fabricación, envasado y empaque, almacenamiento de producto terminado, condiciones de transporte.

6. Acceso a los servicios de análisis de calidad del producto: se observa si la planta cuenta con servicios o tiene convenio algún laboratorio.

Los resultados de este diagnóstico Agroacecar SAS fueron los siguientes: 1) Aseguramiento y control de calidad, 72\%; 2) Instalaciones físicas, $79 \%$; 3) Instalaciones sanitarias, 84\%; 4) Personal manipulador de alimentos, $78 \%$ que incluye: prácticas higiénicas y medidas de protección, 90\% y educación y capacitación, 65\%; 5) Condiciones de saneamiento, 74\%; que incluye: abastecimiento de agua potable, 64\%; manejo de residuos líquidos, 80\%; manejo y disposición de residuos sólidos, 88\%; limpieza y desinfección, 70\% y control de plagas (artrópodos, roedores, aves) 68\% ; 6) Condiciones de proceso y fabricación, $80 \%$ que incluye: equipos y utensilios, $74 \%$; higiene locativa de la sala de proceso, 
72\%; materias primas e insumos; $70 \%$; envases, $93 \%$; operaciones de fabricación, $84 \%$; operaciones de envasado y empaque, $80 \%$; almacenamiento de producto terminado, $70 \%$ y condiciones de transporte, 97\%; 7) Acceso a los servicios de laboratorio, $50 \%$ con un porcentaje de cumplimiento de $74 \%$.

El aseguramiento y control de la calidad (Figura 2), el 60\% de los parámetros evaluados cumple parcialmente, mientras que el $40 \%$ no cumple con las disposiciones establecidas de acuerdo a la normatividad, dentro de estas actividades se incluye que la empresa no tiene establecida una política de calidad, no existen manuales, guías o instructivos, sobre equipos, proceso, condiciones de almacenamiento y distribución. Con relación a las instalaciones el $29 \%$ de los parámetros evaluados cumplen completamente, el $36 \%$ son de manera parcialmente y el $36 \%$ no cumple, las no conformidades identificadas son referentes a la construcción ya que no se presenta la suficiente protección contra la entrada de plagas, polvo, lluvia, no existe una separación física clara entre las áreas de la planta, no se encuentran identificadas las tuberías, no hay una adecuada señalización, circulación de personas salidas de emergencia. De acuerdo a la Figura 3 , se puede observar que el $40 \%$ de los parámetros evaluados en instalaciones sanitarias cumple completamente, el $40 \%$ parcialmente y el $20 \%$ no cumple, las no conformidades identificadas es que la planta no cuenta con área social, hay vestieres y casilleros, pero no están identificadas por sexo, y se encuentran dentro de la misma unidad de construcción junto con los sanitarios.

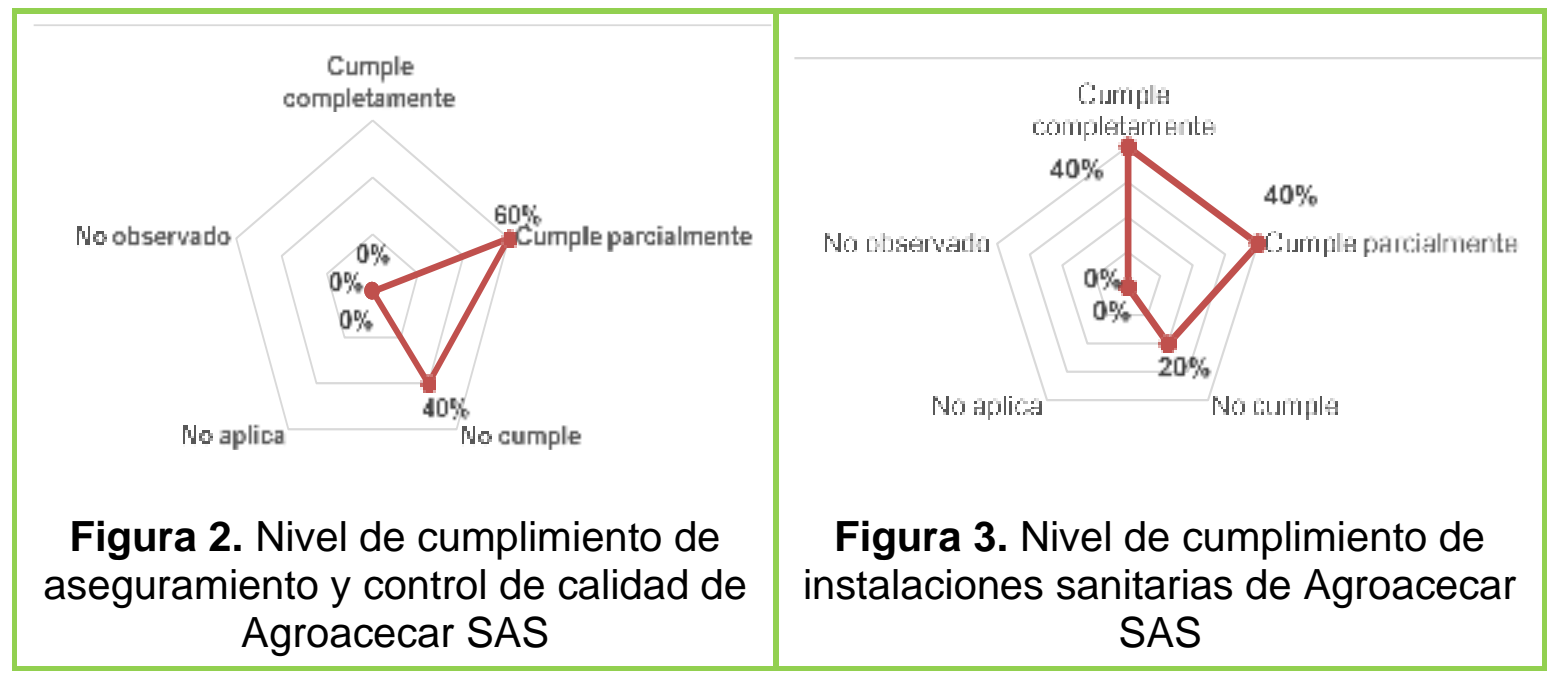


Con relación al personal que manipula los alimentos, en la Figura 4 se puede observar que el $67 \%$ de los aspectos evaluados cumple completamente, el $17 \%$ cumple parcialmente y el $17 \%$ no cumple con los requerimientos, es decir, que el personal manipulador no está utilizando los elementos de protección adecuados para sus actividades de operación. En los resultados de capacitación y educación de personal, se observó que solo el $25 \%$ de los parámetros cumple parcialmente y el $75 \%$ no cumple, esto se debe a que en la empresa no existe un programa en este sentido en la planta, tampoco existen avisos alusivos a prácticas higiénicas, medidas de seguridad, ubicación de extintores, entre otras cosas. No se llevan registros de capacitación en manipulación de alimentos.

En las condiciones de saneamiento (Figura 5), se puede apreciar que el $11 \%$ de los requisitos se cumplen completamente, el $22 \%$ de manera parcial, el $44 \%$ no cumple y el $22 \%$ no aplica. Esto quiere decir que las no conformidades se deben a la falta de procedimientos sobre el manejo y calidad de agua, así mismo, no se llevan los registros de laboratorio, ni control de cloro residual, adicionalmente, no cuentan con un el debido soporte que garantice que el agua utilizada en la planta sea potable. Por otra parte, los puntos que no aplican se deben a que la planta no requiere de hielo y por ende no requiere procesos para esta operación. En el manejo y disposición de residuos líquidos el 100\% de los parámetros evaluados cumplen parcialmente, esto se debe a que, no se existen documentos que soporten que se está garantizando un adecuado manejo de estos residuos en la empresa. Con respecto al manejo de residuos sólidos el $40 \%$ de los requerimientos cumple completamente y el $60 \%$ cumple parcialmente; esto se debe a que no existen documentos que garanticen la adecuada disposición de los residuos sólidos. En actividades de limpieza y desinfección el $50 \%$ se cumple parcialmente los requisitos, principalmente se identificó que la empresa no cuenta con procedimientos para realizar estas labores y tampoco lleva registros de las actividades correspondientes.

Respecto al control de plagas y enfermedades, el $40 \%$ de los parámetros evaluados cumplen parcialmente y el $60 \%$ no cumple, se debe a que no existen documentos que garanticen que se está realizando un control de plagas adecuado. 


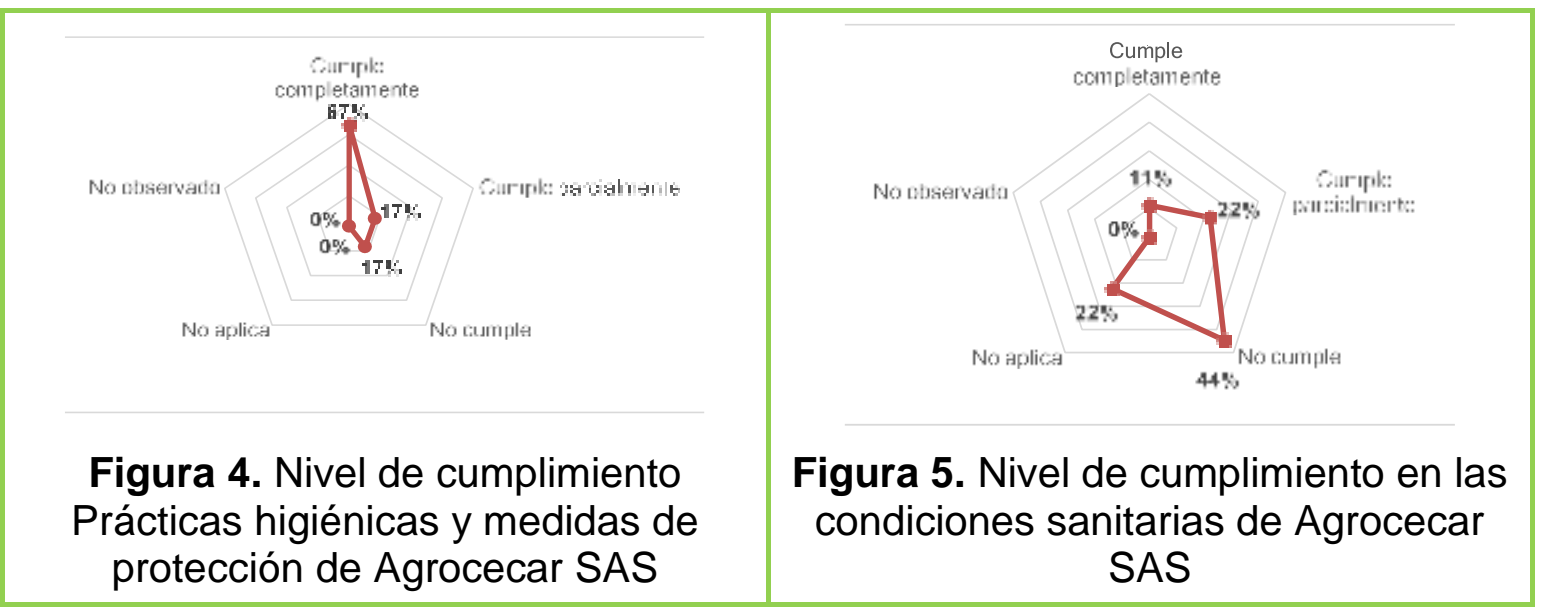

En las condiciones del proceso y fabricación el mantenimiento de equipos y utensilios, el $29 \%$ de los requisitos cumple completamente, el otro $29 \%$ parcialmente, el $24 \%$ no cumple y el $18 \%$ no aplica. Se identificó, que la empresa no cuenta con planes o procedimientos de calibración y mantenimiento de equipos por tanto no se llevan registros, adicionalmente, algunos de los utensilios que se manipulan no cumplen con las condiciones sanitarias adecuadas y algunos de los equipos como los hornos no cuentan con equipos de medición para temperatura. Finalmente, los requerimientos que no aplican porque la empresa no cuenta con los equipos correspondientes. En higiene locativa de la sala de proceso se observa un $18 \%$ de cumplimiento total de los requerimientos, el 27\% cumple parcialmente el $50 \%$ no cumple y el $5 \%$ no aplica. Se identificó que la empresa no cuenta con las paredes y techos adecuados para la higiene, las ventanas presentaban suciedad, en las uniones del piso y la pared se presentó suciedad, en algunos cuartos se evidencio suciedad. El control de materias primas e insumos (figura 6) se puede observar que el $10 \%$ de los parámetros evaluados se cumplen completamente, el $30 \%$ se cumple parcialmente, el $60 \%$ no cumple, las no conformidades identificadas se deben a que no existe como documentos del control de materias primas e insumos, no hay fichas técnicas, no se lleva el seguimiento de control de calidad a materias primas e insumos, y los productos no están rotulados de acuerdo a la normatividad exigida. En los aspectos evaluados en envases, el $67 \%$ cumple y el 
$33 \%$ parcialmente, las faltas encontradas en este aspecto fueron que aquellos envases no son inspeccionados al momento de recibirlos.

Referente a las operaciones de fabricación, el $40 \%$ de los parámetros evaluados cumplen completamente, el otro $40 \%$ cumple parcialmente, y el $20 \%$ no cumple. Se identificó que no se realizan controles a las operaciones críticas del proceso, por ende, no pueden garantizar la inocuidad del producto, adicionalmente, el proceso no lleva orden lineal y secuencial por tanto se puede correr el riesgo de contaminación cruzada. Referente a las condiciones de empaque, el 33\% cumple completamente, el otro $33 \%$ cumple parcialmente y finalmente no cumple el $33 \%$, se identificó que las no conformidades están asociadas a que no se lleva registro de elaboración y producción, tampoco se están rotulando los productos, acorde a la normatividad vigente.

En el almacenamiento de producto terminado, el $50 \%$ de los parámetros evaluados se cumplen parcialmente y el otro $50 \%$ no cumple, se identificó que no se registran las condiciones de almacenamiento no se tiene control de entrada, salida y rotación de productos, los productos que son devueltos a la fábrica por cualquier condición no se están controlando y tampoco se cuenta un espacio para el manejo adecuado de estos y en las condiciones de transporte, el $83 \%$ cumple completamente y el $17 \%$ no aplica, las condiciones de transporte son adecuadas, sin embargo, la empresa desea incluir un seguimiento de control. En el acceso a los servicios de laboratorio se observó que el $50 \%$ no cumple y el otro $50 \%$ no aplica, se identificó que la empresa no cuenta con espacio y recurso para tener laboratorio propio y tampoco tiene convenio con laboratorios externos.

Se observó un nivel de cumplimiento global de $74 \%$ teniendo en cuenta las disposiciones vigentes relacionadas en la Tabla 1.

\section{Recomendaciones para implementar las BPM en Agroacecar SAS}

De acuerdo con los resultados de diagnóstico se elaboró la categorización para poder establecer prioridades en el plan de mejora de la siguiente manera: 1) Nivel crítico, $60 \%$ a $70 \%$ : hace referencia a los aspectos con mayor prioridad para mejorar 
como: educación y capacitación, abastecimiento de agua potable, limpieza y desinfección, control de plagas, materias primas e insumos, almacenamiento de producto terminado y acceso a los servicios de laboratorio. 2) Nivel moderado, $71 \%$ a $80 \%$ : hace referencia a los aspectos como: aseguramiento y control de calidad, Instalaciones físicas, Manejo de residuos líquidos, equipos y utensilios, higiene locativa de la sala de procesos, operaciones de envasado y empaque. 3) Nivel intermedio, $81 \%$ a $95 \%$ : representa los aspectos con un nivel bajo de prioridad como: instalaciones sanitarias, prácticas higiénicas y medidas de protección, manejo de residuos sólidos, envases, operaciones de fabricación, condiciones de transporte.

Con estos resultados se elaboró para la empresa un manual de Buenas Prácticas de manufactura (BPM), pues este documento sería de apoyo para organizar todas las actividades de la empresa y se estructuró con base a los requisitos establecidos en la Resolución 2674 de 2013, con el objetivo de reducir al mínimo los riesgos de contaminación biológica, química o física, que pueden ocurrir durante el procesamiento de alimentos, En este manual se describe las áreas de aplicación y funcionamiento de la siguiente manera: Capítulo 1: Edificación e instalaciones: condiciones generales de localización y accesos como diseño, construcción, abastecimiento de agua, disposición de residuos, instalaciones sanitarias, teniendo en cuenta los pisos, drenajes, paredes, techos, ventanas, otras aberturas, puertas, iluminación y ventilación. Capítulo 2: Equipos y utensilios. Capítulo 3: Personal manipulador de alimentos teniendo en cuenta: estado de salud, educación y capacitación y prácticas higiénicas con medidas de protección. Capítulo 4: Requisitos higiénicos de fabricación en materias primas e insumos, envases y embalajes y proceso de fabricación. Capítulo 5: aseguramiento de la calidad en el cual se incluye: control de calidad e inocuidad, Sistema de control y laboratorios. Capítulo 6: Saneamiento. Capítulo 7: Almacenamiento, distribución y transporte. La documentación perteneciente al manual de Buenas Prácticas de Manufactura (Figura 7), se conformó de acuerdo a unos parámetros establecidos para lograr uniformidad dentro de cada uno de los documentos. 


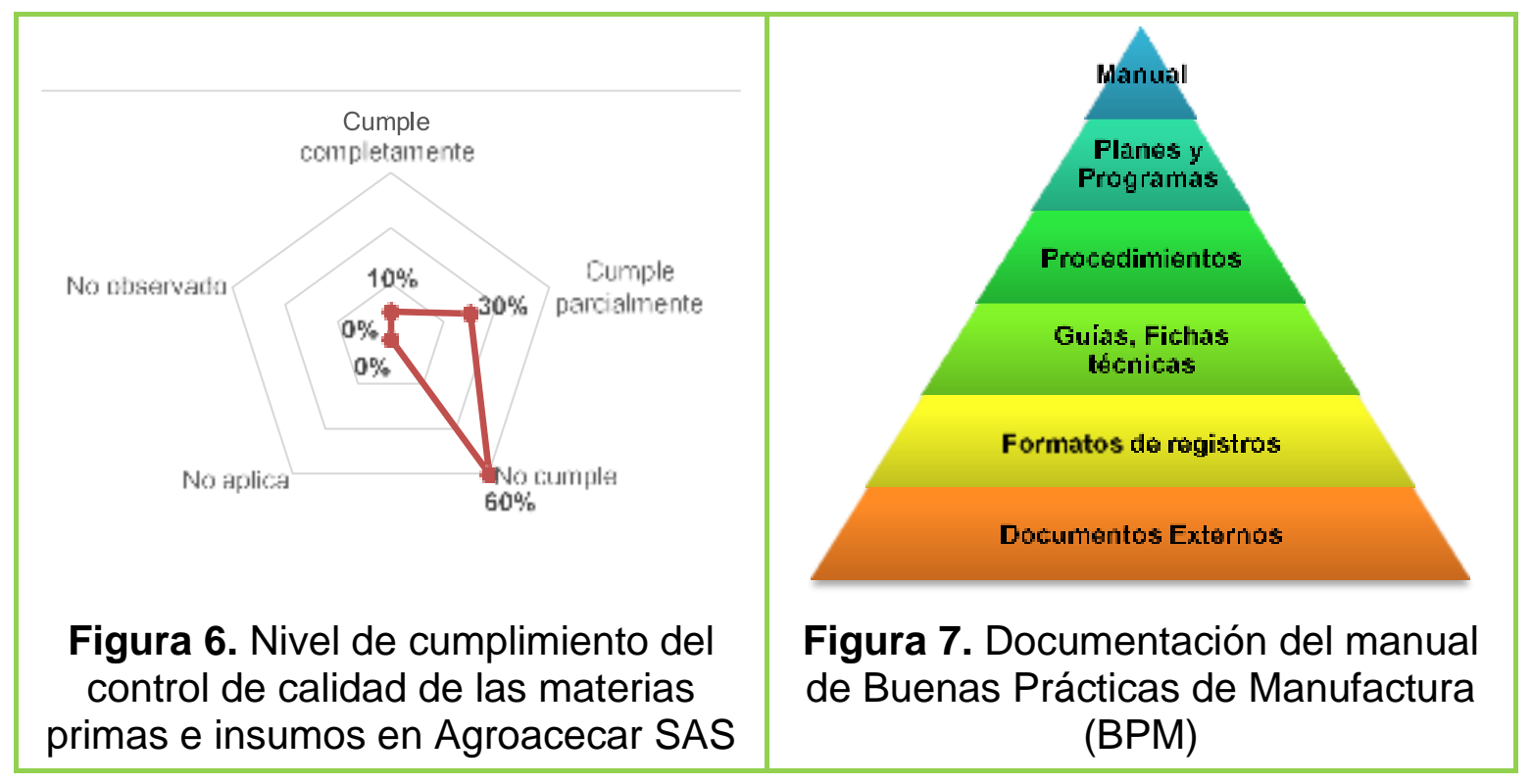

\section{CONCLUSIONES}

Se realizó el diagnóstico de las condiciones higiénico-sanitarias a la planta de Agroacecar SAS donde se obtuvo un porcentaje del $74 \%$, con base en estos resultados se evidenció que la empresa no cuenta con un sistema documental de Buenas Prácticas de Manufactura.

Se diseñó el Manual de Buenas Prácticas de Manufactura estableciendo las prácticas de higiene en la manipulación y elaboración de alimentos, basado en la Resolución 2674 de 2013.

Se digitalizó el Manual de Buenas Prácticas de Manufactura con acceso remoto "https://diegoguzman13.wixsite.com/misitio" con su respectiva documentación ajustada a las necesidades de la empresa Agroacecar SAS.

Se elaboró un total de 1 manual, 9 programas, 1 plan, 24 formatos de registros, 8 fichas técnicas y 2 instructivos, completando la documentación requerida por la Resolución 2674 de 2013, para un total de 51 documentos.

Se realizó una propuesta de implementación para la empresa Agroacecar SAS, determinado los tiempos de ejecución de cada una de las fases propuestas para 
que a empresa pueda dar cumplimento a los requisitos mínimos exigidas por la legislación colombiana.

\section{RECOMENDACIONES}

Se recomienda que la empresa mantenga el mejoramiento continuo del sistema de Buenas Prácticas de Manufactura, con el fin de que ayude a incrementar la productividad y a la vez ser más competitivos en el mercado que les compete.

Realizar capacitaciones continuas a los empleados de acuerdo al plan de capacitación del 2021

Socializar e implementar el manual de Buenas Prácticas de Manufactura y toda su documentación con el fin de enriquecer el conocimiento y la obligación que conlleva manipular alimentos dándole así una vital importancia a la inocuidad alimentaria.

Se recomienda que los directivos tengan la responsabilidad de ejecutar el plan de implementación propuesto para su organización y de controlar su ejecución y cumplimiento.

Se recomienda realizar auditorías de verificación para asegurar que se esté cumpliendo con toda la documentación e implementación de los documentos elaborados.

Actualizar el Manual de Buenas Prácticas de Manufactura conforme se vayan realizando las modificaciones o cambios de infraestructura e implementación de registros y procedimientos para llevar una documentación global más actualizada.

\section{REFERENCIAS BIBLIOGRÁFICAS}

1. Albarracín $F$, Carrascal A. Manual de buenas prácticas de manufactura para microempresas lácteas. Pontificia Universidad Javeriana, 179 p. 2005.

2. Ayuso G, Castillo M. Globalización y nostalgia. Cambios en la alimentación de las familias yucatecas. Rev. Alimentación contemporánea y desarrollo regional, $27 \quad$ (50): 1-28. 2017.2 Disponible En: https://www.redalyc.org/jatsRepo/417/41751187007/41751187007.pdf

3. Comisión del Codex Alimentarius (CCA). Manual de Procedimiento Programa Conjunto FAO/OMS sobre Normas Alimentarias. 23르 ed. 243 p. 2015. Disponible En: http://www.fao.org/3/i4354s/i4354s.pdf 
4. Congreso de Colombia (CC). Ley 9 (24 de enero 1979). Por lo cual establece medidas sanitarias Bogotá D.C. 1979. Disponible En: http://copaso.upbbga.edu.co/legislacion/ley 9 1979.Codigo\%20Sanitario\%20 Nacional.pdf

5. Institución Nacional Penitenciario y Carcelario (INPEC). Manual de buenas prácticas de manufactura: Panadería EPMSC CALI., M-PT-001. 2017. Disponible https://red.uao.edu.co/bitstream/handle/10614/9817/T7485A.pdf?sequence $=2$ \&isAllowed $=y$

6. International Dynamic Advisors (INTEDYA). Área de Seguridad Alimentaria. Buenas Prácticas de Manufactura, BPM. 30 p. Disponible En: https://www.intedya.com/productos/seguridad\%20alimentaria/BPM/PIC BPM.p df

7. Mena, M. Prerrequisitos y Sistema HACCP en la Industria Alimentaria. Tesis Nutricionista. España, 33 p. 2014. En: https://1library.co/document/zkwr634zprerrequisitos-y-sistema-haccp-en-la-industria-alimentaria.html

8. Ministerio de Salud y Protección Social (MSPS) y Ministerio de Ambiente, Vivienda y Desarrollo Territorial (MAVDT). Resolución 2115 (22 de junio de 2007). Por medio de la cual se señalan características, instrumentos básicos y frecuencias del sistema de control y vigilancia para la calidad del agua para consumo humano. 2007.2 Disponible En: https://www.minambiente.gov.co/images/GestionIntegraldelRecursoHidrico/pdf Legislaci\%C3\%B3n del agua/Resoluci\%C3\%B3n 2115.pdf

9. Ministerio de Salud y Protección Social. (MSPS). Resolución 5109 (29 de diciembre de 2005). Por la cual se establece el reglamento técnico sobre los requisitos de rotulado o etiquetado que deben cumplir los alimentos envasados y materias primas de alimentos para consumo humano. 2005. Disponible En: https://scj.gov.co/es/transparencia/marcolegal/normatividad/resoluci\%C3\%B3n-5109-2005

10. Ministerio de Salud y Protección Social. (MSPS). Decreto 1575 (9 de mayo de 2007). Por el cual se establece el Sistema para la Protección y Control de la Calidad del Agua para Consumo Humano. 2007. Disponible En: https://www.minambiente.gov.co/images/GestionIntegraldelRecursoHidrico/pdf Disponibilidad-del-recurso-hidrico/Decreto-1575-de-2007.pdf

11. Ministerio de Salud y Protección Social (MSPS). Resolución 333 (10 de febrero de 2011). Por la cual se establece el reglamento técnico sobre los requisitos de rotulado o etiquetado nutricional que deben cumplir los alimentos envasados para consumo humano. 2011. Disponible En: https://extranet.who.int/nutrition/gina/sites/default/filesstore/COL\%202011\%20 RESOLUCl\%C3\%93N\%200333\%20\%28etiquetado\%20nutricional\%29.pdfBog otá

12. Ministerio de Salud y Protección Social (MSPS). Resolución 683 (28 de marzo de 2012). Por medio de la cual se expide el Reglamento Técnico sobre los requisitos sanitarios que deben cumplir los materiales, objetos, envases y equipamientos destinados a entrar en contacto con alimentos y bebidas para consumo humano. $2012 . \quad$ Disponible 
https://www.invima.gov.co/documents/20143/437174/2RESOLUCION 683 DE 2012.pdf/6d837632-bce1-c26a-70ce-28da9665f788

13. Ministerio de Salud y Protección Social (MSPS). Resolución 4142 (12 de diciembre de 2012). Por la cual se establece el reglamento técnico sobre los requisitos sanitarios que deben cumplir los materiales, objetos, envases y equipamientos metálicos destinados a entrar en contacto con alimentos y bebidas para consumo humano en el territorio nacional. 2012. Disponible En: http://www.suin-juriscol.gov.co/viewDocument.asp?ruta=Resolucion/30033907

14. Ministerio de Salud y Protección Social (MSPS). Resolución 835 (23 de marzo de 2013). Por medio de la cual se expide el Reglamento Técnico sobre los requisitos sanitarios que deben cumplir los materiales, objetos, envases y equipamientos destinados a entrar en contacto con alimentos y bebidas para consumo humano. 2013. Disponible En: http://www.suinjuriscol.gov.co/viewDocument.asp?ruta=Resolucion/30033912

15. Ministerio de salud y protección social, (MSPS). Resolución 2674 (22, julio 2013). Por la cual se reglamenta el artículo 126 del Decreto-ley 019 de 2012 y se dictan otras disposiciones. 2013. Disponible En: http://extwprlegs1.fao.org/docs/pdf/col145241.pdf

16. Ministerio de Salud y Protección Social (MSPS). Resolución 3929 (2 de octubre de 2013). Por la cual se establece el reglamento técnico sobre los requisitos sanitarios que deben cumplir las frutas y las bebidas con adición de jugo (zumo) o pulpa de fruta o concentrados de fruta, clarificados o no, o la mezcla de estos que se procesen, empaquen, transporten, importen y comercialicen en el territorio nacional. 2013.2 Disponible https://www.invima.gov.co/documents/20143/441425/Resolucion-39292013.pdf/28252dd6-41eb-a575-8ec4-c876e6326a5e

17. Ministerio de Salud y Protección Social, (MSPS). Resolución 719 (11, marzo 2015). Por la cual se establece la clasificación de alimentos para consumo humano de acuerdo con el riesgo en salud pública. 20 p. 2015. Disponible En: https://www.invima.gov.co/documents/20143/344791/resoluci\%C3\%B3n0719d e2015anexot\%C3\%A9cnico.pdf

18. Organización de las Naciones Unidas para la Alimentación y la Agricultura (FAO). Buenas manufacturas prácticas de en la elaboración de productos lácteos FAO Guatemala. 28 p. 2011. Disponible En: http://www.fao.org/3/bo953s/bo953s.pdf

19. Rodríguez H, Barreto G, Sedrés M, Bertot J, Martínez S, Guevara G. Las enfermedades transmitidas por alimentos, un problema sanitario que hereda $\mathrm{e}$ incrementa el nuevo milenio. Rev. Electrónica Veterinaria, 16 (8): 1-27. 2015. Disponible En: https://www.redalyc.org/pdf/636/63641401002.pdf

20. Ruiz A. Muestreos de Aceptación. Apuntes de Clase. Comillas. Madrid. 41 p. 2006. Disponible En: https://web.cortland.edu/matresearch/aceptacion.pdf 